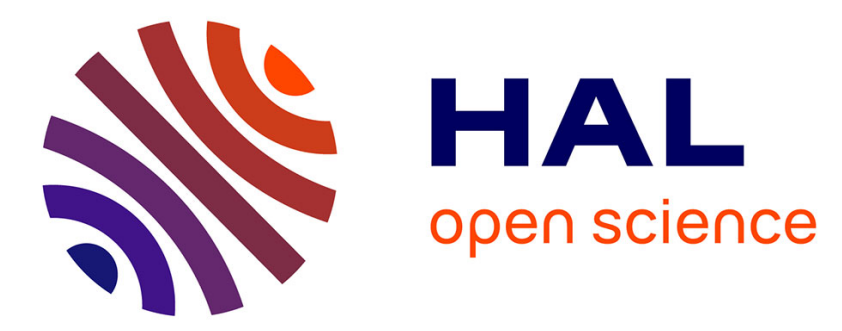

\title{
Guiding-center simulations on curvilinear meshes
}

Jean-Philippe Braeunig, Nicolas Crouseilles, Michel Mehrenberger, Eric

Sonnendrücker

\section{To cite this version:}

Jean-Philippe Braeunig, Nicolas Crouseilles, Michel Mehrenberger, Eric Sonnendrücker. Guidingcenter simulations on curvilinear meshes. Numerical Models for Controlled Fusion (NMCF09), 2009, Porquerolles, France. pp.271-282, 10.3934/dcdss.2012.5.271 . hal-01298727

\section{HAL Id: hal-01298727 \\ https://hal.science/hal-01298727}

Submitted on 6 Apr 2016

HAL is a multi-disciplinary open access archive for the deposit and dissemination of scientific research documents, whether they are published or not. The documents may come from teaching and research institutions in France or abroad, or from public or private research centers.
L'archive ouverte pluridisciplinaire $\mathbf{H A L}$, est destinée au dépôt et à la diffusion de documents scientifiques de niveau recherche, publiés ou non, émanant des établissements d'enseignement et de recherche français ou étrangers, des laboratoires publics ou privés. 


\title{
Guiding-Center simulations on curvilinear meshes.
}

\author{
J.-P. Braeunig ${ }^{1} 2{ }^{3}$, Nicolas Crouseilles ${ }^{12}$, \\ Michel Mehrenberger ${ }^{2}$, ERIC SonnendrüCKer ${ }^{2}{ }^{1}$.
}

\begin{abstract}
The purpose of this work is to design simulation tools for magnetised plasmas in the ITER project framework. The model addressed to simulate turbulent transport in a Tokamak is a 5D gyrokinetic model, taking advantage of the particular motion of particles due to the presence of a strong magnetic field. Accurate schemes and parallel algorithms are designed to bear these heavy simulations. One step further for the computational accuracy is to define a mesh aligned with magnetic lines. In this way, we first study algorithms and schemes in curvilinear coordinates for a reduced Vlasov-Poisson model: the 2D Guiding-Center model. A first method is described and numerical results are presented to show its good properties.
\end{abstract}

\section{Introduction}

In a Tokamak the plasma is kept out of the vessel walls by a magnetic field which lines have a specific helicoidal geometry. Turbulence develops in the plasma and leads to thermal transport which decreases the confinement efficiency. The characteristic mean free path is large, even compared with the vessel size, therefore a kinetic description of particles is required, see Dimits [4]. Therefore, one should use a 6D Vlasov-Poisson like model for both ions and electrons to properly describe the plasma evolution. However, the plasma flow in the presence of a strong magnetic field has characteristics that allow some physical assumptions to reduce the model, see Brizard and Hahm [1]. As a consequence, the 6D Vlasov-Poisson model is approximated by a 5D gyrokinetic model by averaging equations in such a way the $6 \mathrm{D}$ toroidal coordinates system $\left(r, \theta, \phi, v_{r}, v_{\theta}, v_{\phi}\right)$ becomes a $5 \mathrm{D}$ coordinates system $\left(r, \theta, \phi, v_{\|}, \mu\right)$, with $v_{\|}$the parallel to the field lines component of the velocity and $\mu=m v_{\perp}^{2} / 2 B$ the adiabatic invariant which depends on the norm of the perpendicular to the field lines components of the velocity $v_{\perp}^{2}$, on the magnetic field magnitude $B$ and on the particles mass $m$. Moreover, the electrons are assumed to be at equilibrium, i.e. the effect of the electrons

\footnotetext{
${ }^{1}$ INRIA Nancy-Grand Est, 615 rue du Jardin Botanique, 54600 Villers-lès-Nancy

${ }^{2}$ IRMA, Université de Strasbourg, 7 rue René-Descartes, 67084 Strasbourg Cedex

${ }^{3} \mathrm{CEA} / \mathrm{DIF}$ Bruyères-le-Châtel, 91297 Arpajon Cedex
} 
cyclotronic motion is neglected. Their distribution is then supposed to be constant in time. The 5D gyrokinetic model then reduces to a Vlasov like equation for ions guiding-center motion:

$$
\frac{\partial \bar{f}_{\mu}}{\partial t}+\frac{d X}{d t} \cdot \nabla_{X} \bar{f}_{\mu}+\frac{d v_{\|}}{d t} \partial_{v_{\|}} \bar{f}_{\mu}=0
$$

where $\bar{f}_{\mu}\left(X, v_{\|}\right)$is the ion distribution function with $X=(r, \theta, \phi)$, velocity $d X / d t$ and acceleration $d v_{\|} / d t$ define the guiding-center trajectories.

If $\nabla_{\left(X, v_{\|}\right)} \cdot\left(d X / d t, d v_{\|} / d t\right)^{t}=0$ then the model is termed as conservative and can be written in an equivalent conservative form:

$$
\frac{\partial \bar{f}_{\mu}}{\partial t}+\nabla_{X} \cdot\left(\frac{d X}{d t} \bar{f}_{\mu}\right)+\partial_{v_{\|}}\left(\frac{d v_{\|}}{d t} \bar{f}_{\mu}\right)=0
$$

This equation for ions is coupled with a quasi-neutrality equation for the electric potential $\Phi(R)$ on particles position, with $R=X-\rho_{L}$ (with $\rho_{L}$ the Larmor radius):

$$
-\frac{1}{B \omega_{i}} \nabla_{\perp} \cdot\left(n_{e} \nabla_{\perp} \Phi\right)+\frac{e}{\kappa T_{e}}\left(\Phi-<\Phi>_{\theta, \phi}\right)=\int J_{0}\left(\bar{f}_{\mu}\right) d \mu d v_{\|}-n_{e}
$$

where $J_{0}$ is the gyroaverage operator, $n_{e}$ is an equilibrium electron density, $T_{e}$ the electron temperature, $e$ the electron charge, $\kappa$ the Boltzmann constant for electrons and $\omega_{i}$ the cyclotron frequency for ions. The operator $\nabla_{\perp}$ denotes the gradient in the directions perpendicular to the magnetic field lines.

These equations are of a simple form, but they have to be solved very efficiently because of the 5D space and the large characteristic time scales considered. Particles velocity in the parallel to the magnetic field lines direction is much higher than in the perpendicular directions. Particles motion is then strongly anisotropic. In the GYSELA code, the mesh is currently structured and based on toroidal coordinates. This is not well adapted to the flow because of the magnetic field lines curvature. Therefore, we want to use curvilinear coordinates in such a way mesh lines are aligned with the magnetic field lines, to better capture the anisotropy of the system and avoid numerical diffusion in the perpendicular directions, see Brizard and Hahm [1] for the foundations of this work. This adapted discretization of the gyrokinetic model should permit to strongly reduce the number of cells in the parallel to the magnetic field lines direction.

On the way of this curvilinear discretization of the gyrokinetic model, we first worked on the the so-called Guiding-Center model, which is a reduced 
2D Vlasov equation coupled with a Poisson equation:

$$
\left\{\begin{array}{l}
\frac{\partial f}{\partial t}+E_{\perp} \cdot \nabla_{X} f=0 \\
-\Delta \Phi=f
\end{array}\right.
$$

where $f(x, y)$ is the particles distribution function with $X=(x, y), d X / d t=$ $E_{\perp}=\left(E_{y},-E_{x}\right)^{t}$ define the trajectories with $E=\left(E_{x}, E_{y}\right)^{t}=-\nabla_{X} \Phi$ and $\Phi(x, y)$ the electric potential. The model is conservative since $\nabla_{X} \cdot E_{\perp}=0$. This simple model contains some features of the gyrokinetic model and have turbulent unstable modes which growth rate can be computed analytically.

To enforce the strict mass conservation in the system, one wants to discretize the Guiding-Center model in its conservative form. It also allows properly a directional splitting, which is very convenient to design a parallel MPI algorithm and to deal with curvilinear coordinates without having to handle the aligned mesh geometry. This is possible because the divergence of the advection field is zero:

$$
\nabla_{X} \cdot\left(E_{\perp}\right)=\partial_{x} E_{y}-\partial_{y} E_{x}=-\partial_{x} \partial_{y} \Phi+\partial_{x} \partial_{y} \Phi=0 .
$$

Therefore, the system (4) is strictly equivalent to the following:

$$
\begin{aligned}
& \frac{\partial f}{\partial t}+\nabla_{X} \cdot\left(E_{\perp} f\right)=0, \\
& -\Delta \Phi=f .
\end{aligned}
$$

This conservative system will be discretized using a conservative semiLagrangian scheme, the Parabolic Spline Method (PSM, see Zerroukat et al [11] [12] and Crouseilles, Mehrenberger, Sonnendrücker [2]) scheme. It is a fourth order scheme which is equivalent for linear advection to the Backward Semi-Lagrangian Scheme (BSL, see Cheng and Knorr [3] and Sonnendrücker et al [10]) with a cubic B-splines interpolation operator, but in a conservative form. The Vlasov equation will be discretized using a directional splitting solving $D$ (dimension of space) 1D conservative equations with the PSM scheme. This allows to handle easily on structured grids any curvilinear coordinates system. The Poisson equation will be solved on a cartesian grid and the electric potential is remapped on the curvilinear mesh. This choice is made to decouple the development of curvilinear coordinates for the Vlasov equation (1) and for the quasi-neutrality equation (3), which the latest is harder to handle for this change of coordinates.

In this paper, the PSM scheme will be detailed. The next section is dedicated 
to the writing of usual differential operators and in particular the Vlasov equation in curvilinear coordinates. At last some numerical results will be shown.

\section{The conservative Parabolic Spline Method (PSM) for Vlasov equations}

The PSM scheme is very similar to the BSL one (see [3][10]), because it is also based on characteristic curves of the flow and on a cubic spline reconstruction of the distribution function. These schemes are exactly the same for linear advection. However, the PSM scheme is based on the conservative form of Vlasov equations and provides a perfect mass conservation when dealing with non-linear advection, which the BSL scheme do not.

Let us consider a Vlasov equation in its conservative form:

$$
\frac{\partial f}{\partial t}+\nabla_{x} \cdot(a f)=0
$$

with $f(x, t)$ a scalar function, position $x \in \mathbb{R}^{D}$ and $a(x, t) \in \mathbb{R}^{D}$ the advection field. The mass $m$ conservation in a Lagrangian volume reads:

$$
m=\int_{V o l^{n+1}} f\left(x, t^{n+1}\right) d \Omega=\int_{V o l^{n}} f\left(x, t^{n}\right) d \Omega,
$$

with the characteristic curves $X$ defined as $\frac{d X(x, t)}{d t}=a(x, t)$ and $x^{n}=$ $X\left(x^{n}, t^{n}\right)$, and the volume $V_{o l} l^{n}=\left\{X\left(x^{n+1}, t^{n}\right)\right.$ such that $X\left(x^{n+1}, t^{n+1}\right) \in$ $\left.V o l^{n+1}\right\}$ defines the Lagrangian motion of $V o l^{n}$ by the field $a(x, t)$.

This conservative formalism properly allows a directional splitting without loosing the mass conservation, because each 1D step will be written in a conservative form. From a numerical point of view, the 1D advection for each direction will be approximated by the following $1 D$ equation:

$$
\int_{x_{i-1 / 2}^{n+1}}^{x_{i+1 / 2}^{n+1}} f\left(x, t^{n+1}\right) d x=\int_{x_{i-1 / 2}^{n}}^{x_{i+1 / 2}^{n}} f\left(x, t^{n}\right) d x,
$$

with $x_{i+1 / 2}^{n+1}=X\left(x_{i+1 / 2}^{n+1}, t^{n+1}\right)$ settled as the $1 \mathrm{D}$ mesh nodes and $x_{i+1 / 2}^{n}=$ $X\left(x_{i+1 / 2}^{n+1}, t^{n}\right)$ the so called characteristic "foot" of $x_{i+1 / 2}^{n+1}$. Let us define the average of $f$ in cell $i$ at time $t^{n+1}$ :

$$
\bar{f}_{i}^{n+1}=\frac{1}{\Delta x} \int_{x_{i-1 / 2}^{n+1}}^{x_{i+1 / 2}^{n+1}} f\left(x, t^{n+1}\right) d x
$$


and the primitive function

$$
F^{n}(z)=\int_{x_{1 / 2}}^{z} f\left(x, t^{n}\right) d x
$$

with $x_{1 / 2}$ an arbitrary reference point of the domain and for instance the first node of the grid $\left\{x_{i-1 / 2}\right\}_{i=1, N+1}$.

Therefore, we have to solve a nonlinear system to obtain $X\left(x_{i+1 / 2}^{n+1}, t^{n}\right)=$ $x_{i+1 / 2}^{n}$ (which is similar with the BSL one) to obtain a solution of equation (8) that simply writes:

$$
\frac{X\left(x_{i+1 / 2}^{n+1}, t^{n+1}\right)-X\left(x_{i+1 / 2}^{n+1}, t^{n}\right)}{\Delta t}=a\left(X_{i+1 / 2}^{n+1 / 2}, t^{n+1 / 2}\right),
$$

with

$$
\begin{aligned}
& X_{i+1 / 2}^{n+1 / 2}=\left(X\left(x_{i+1 / 2}^{n+1}, t^{n+1}\right)+X\left(x_{i+1 / 2}^{n+1}, t^{n}\right)\right) / 2, \\
& \bar{f}_{i}^{n+1} \Delta x=F^{n}\left(X\left(x_{i+1 / 2}^{n+1}, t^{n}\right)\right)-F^{n}\left(X\left(x_{i-1 / 2}^{n+1}, t^{n}\right)\right),
\end{aligned}
$$

with the time step $\Delta t=t^{n+1}-t^{n}$ and the space step $\Delta x=x_{i+1 / 2}^{n+1}-x_{i-1 / 2}^{n+1}$. The scheme is constituted with two steps:

- For a mesh node $i+1 / 2$ which location is settled as $x_{i+1 / 2}^{n+1}=X\left(x_{i+1 / 2}^{n+1}, t^{n+1}\right)$, we have to follow backward the characteristic curve to find the "foot" $x_{i+1 / 2}^{n}=X\left(x_{i+1 / 2}^{n+1}, t^{n}\right)$.

- Computation of the primitive function at mesh nodes $x_{i+1 / 2}^{n+1}$, what is a simple addition because for any $i \in[1, N]$ :

$$
F^{n}\left(x_{i+1 / 2}^{n+1}\right)-F^{n}\left(x_{1 / 2}\right)=\sum_{k=1}^{i} \bar{f}_{k}^{n} \Delta x
$$

then interpolation (cubic spline) of the primitive function $F^{n}(z)$ on the domain with nodal values $F^{n}\left(x_{i+1 / 2}^{n+1}\right)$ to obtain its value at $x_{i+1 / 2}^{n}$, which is not a mesh node in general. Thus we have $\bar{f}_{i}^{n+1} \Delta x=F^{n}\left(x_{i+1 / 2}^{n}\right)-F^{n}\left(x_{i-1 / 2}^{n}\right)$.

This scheme is fourth order in space. It is second order in time with a leap-frog or predictor-corrector time integration scheme to compute the advection field $a\left(x, t^{n+/ 2}\right)$ at time $t^{n+1 / 2}$. This scheme is conservative because 
by integrating on the whole domain:

$$
\begin{aligned}
\int_{x_{1 / 2}^{n+1}}^{x_{N+1 / 2}^{n+1}} f\left(x, t^{n+1}\right) d x & =\sum_{k=1}^{N} \bar{f}_{k}^{n+1} \Delta x=F^{n}\left(x_{N+1 / 2}^{n}\right)-F^{n}\left(x_{1 / 2}^{n}\right) \\
& =\int_{x_{1 / 2}^{n}}^{x_{N+1 / 2}^{n}} f\left(x, t^{n}\right) d x .
\end{aligned}
$$

\section{$3 \quad$ Vlasov Equation in curvilinear coordinates}

\subsection{Curvilinear coordinates}

Let us consider a cartesian coordinates system $x=\left(x_{1}, x_{2}, x_{3}\right) \in \mathbb{R}^{3}$. A curvilinear coordinates system is defined with three functions $\xi_{1}(x), \xi_{2}(x), \xi_{3}(x)$ which the Jacobian is

$$
J=\operatorname{det}\left(\begin{array}{lll}
\frac{\partial \xi_{1}}{\partial x_{1}} & \frac{\partial \xi_{1}}{\partial x_{2}} & \frac{\partial \xi_{1}}{\partial x_{3}} \\
\frac{\partial \xi_{2}}{\partial x_{1}} & \frac{\partial \xi_{2}}{\partial x_{2}} & \frac{\partial \xi_{2}}{\partial x_{3}} \\
\frac{\partial \xi_{3}}{\partial x_{1}} & \frac{\partial \xi_{3}}{\partial x_{2}} & \frac{\partial \xi_{3}}{\partial x_{3}}
\end{array}\right)=\nabla \xi_{1} \cdot\left(\nabla \xi_{2} \times \nabla \xi_{3}\right) .
$$

One can build two bases of $\mathbb{R}^{3}$ by using $\xi_{1}(x), \xi_{2}(x), \xi_{3}(x)$ :

$$
\left(\nabla \xi_{1}, \nabla \xi_{2}, \nabla \xi_{3}\right)
$$

and

$$
\left(\nabla \xi_{2} \times \nabla \xi_{3}, \nabla \xi_{3} \times \nabla \xi_{1}, \nabla \xi_{1} \times \nabla \xi_{2}\right) .
$$

A given vector $A \in \mathbb{R}^{3}$ can be expressed at any point in each base as follow:

$$
\begin{aligned}
& A=A_{1} \nabla \xi_{1}+A_{2} \nabla \xi_{2}+A_{3} \nabla \xi_{3}, \\
& A=A^{1} \nabla \xi_{2} \times \nabla \xi_{3}+A^{2} \nabla \xi_{3} \times \nabla \xi_{1}+A^{3} \nabla \xi_{1} \times \nabla \xi_{2} .
\end{aligned}
$$

Coordinates $A_{i}$ (subscript index) are termed as covariant coordinates of $A$ and coordinates $A^{i}$ (superscript index) are termed as contravariant coordinates of $A$. These two coordinates systems are linked by the metric tensor which is a $3 \times 3$ symmetric matrix written $\mathbb{G}$ and defined by

$$
\mathbb{G}^{-1}=\left(\begin{array}{ccc}
\nabla \xi_{1} \cdot \nabla \xi_{1} & \nabla \xi_{1} \cdot \nabla \xi_{2} & \nabla \xi_{1} \cdot \nabla \xi_{3} \\
\nabla \xi_{2} \cdot \nabla \xi_{1} & \nabla \xi_{2} \cdot \nabla \xi_{2} & \nabla \xi_{2} \cdot \nabla \xi_{3} \\
\nabla \xi_{3} \cdot \nabla \xi_{1} & \nabla \xi_{3} \cdot \nabla \xi_{2} & \nabla \xi_{3} \cdot \nabla \xi_{3}
\end{array}\right)
$$

A common convention is to write $g_{i j}$ the elements of matrix $\mathbb{G}$ and $g^{i j}$ those of the inverse matrix $\mathbb{G}^{-1}$. Therefore we have

$$
A_{i}=\sum_{j} g_{i j} A^{j} \text { and } A^{i}=\sum_{j} g^{i j} A_{i}
$$


Remark 1. The metric tensor determinant is linked to the transformation Jacobian $J$ by $\operatorname{det} \mathbb{G}^{-1}=J^{2}$. Let us write $g=\operatorname{det} \mathbb{G}$, thus we have $J=$ $1 / \sqrt{g}$

Contravariant coordinates are obtained by the scalar product of (15) with $\nabla \xi_{i}$ :

$$
J A^{i}=A \cdot \nabla \xi_{i}, \quad \text { thus } A^{i}=\sqrt{g} A \cdot \nabla \xi_{i} .
$$

Usual operators are then written in curvilinear coordinates as follow:

$$
\begin{aligned}
(\nabla \Phi)^{i} & =\sum_{k=1}^{3} g^{i k} \frac{\partial}{\partial \xi_{k}} \Phi \\
\nabla \cdot A & =\frac{1}{\sqrt{g}} \sum_{i=1}^{3} \frac{\partial}{\partial \xi_{i}}\left(A^{i}\right), \\
\Delta \Phi & =\frac{1}{\sqrt{g}} \sum_{i=1}^{3} \sum_{k=1}^{3} \frac{\partial}{\partial \xi_{i}}\left(\sqrt{g} g^{i k} \frac{\partial}{\partial \xi_{k}} \Phi\right) .
\end{aligned}
$$

\subsection{Vlasov equation}

Let us consider again the Vlasov equation in its conservative form in a cartesian coordinates system $x=(x, y, z)$ :

$$
\frac{\partial f}{\partial t}+\nabla_{x} \cdot(a f)=0
$$

with $f(x, t)$ a scalar function, position $x \in \mathbb{R}^{3}$ and $a(x, t) \in \mathbb{R}^{3}$ the advection field. Considering a curvilinear coordinates system $\xi_{1}(x), \xi_{2}(x), \xi_{3}(x)$ with its associated Jacobian $J=1 / \sqrt{g}$, one can write the Vlasov equation in these curvilinear coordinates:

$$
\frac{\partial f}{\partial t}+\frac{1}{\sqrt{g}} \sum_{i=1}^{3} \frac{\partial}{\partial \xi_{i}}\left(a^{i} f\right)=0
$$

with $a^{i}=\sqrt{g} a \cdot \nabla \xi_{i}$.

Notice that the change of coordinates system yields to a very equivalent form of the divergence. Indeed, since the jacobian $\sqrt{g}$ is not time dependent, the divergence writes:

$$
\frac{\partial \tilde{f}}{\partial t}+\sum_{i=1}^{3} \frac{\partial}{\partial \xi_{i}}\left(\tilde{a}^{i} \tilde{f}\right)=0
$$


with $\tilde{f}=\sqrt{g} f$ and $\tilde{a}^{i}=a \cdot \nabla \xi_{i}$.

For instance, in the cylindrical coordinates system:

$$
\begin{aligned}
& x=r \cos \theta, \\
& y=r \sin \theta, \\
& z=\zeta,
\end{aligned}
$$

the Vlasov equation writes:

$$
\frac{\partial f}{\partial t}+\frac{1}{r}\left(\partial_{r}\left(r a^{r} f\right)+\partial_{\theta}\left(r a^{\theta} f\right)+\partial_{\zeta}\left(r a^{\zeta} f\right)\right)=0,
$$

with the Jacobian $\sqrt{g}=r$ and contravariant coordinates $a^{r}=a \cdot \nabla r=$ $a_{x} \cos \theta+a_{y} \sin \theta, a^{\theta}=\left(-a_{x} \sin \theta+a_{y} \cos \theta\right) / r$ and $a^{\zeta}=a_{z}$.

An equivalent form can be written as follow:

$$
\frac{\partial \tilde{f}}{\partial t}+\partial_{r}\left(a^{r} \tilde{f}\right)+\partial_{\theta}\left(a^{\theta} \tilde{f}\right)+\partial_{\zeta}\left(a^{\zeta} \tilde{f}\right)=0
$$

with $\tilde{f}=r f$.

\subsection{Periodic boundary conditions}

One difficulty may arise, when considering a mesh based on a curvilinear coordinates system, with periodic boundary conditions in a cartesian direction. Each mesh line might not connect with itself but with a neighbouring mesh line, see figure 1. This is a specific problem when using curvilinear coordinates: when defining the adapted mesh, we need to make sure that the mesh is still conform. For instance, each node at the domain lower boundary should have a corresponding node at the upper boundary, even if it does not reconnect on the same mesh line.

This will be a restriction in the choice of the adapted mesh in a Tokamak geometry. The helicoidal geometry of magnetic field lines in the poloidal section of the torus have a rotation rate $q(r)$, the safety coefficient, which is not constant but depends on the radial direction. It is not a rational number in general, thus field lines might not reconnect by periodicity in the toroidal direction, so would neither a mesh adapted to field lines. Two solutions will be investigated. First one is to choose an adapted mesh with a rational safety coefficient $\bar{q}_{i}$ close to each real magnetic field lines safety factor $q\left(r_{i}\right)$, in such a way the angle between mesh lines and magnetic field lines will be as small as possible (figure 4 shows that the benefits of adapting the mesh remains for small angles). Second one is suggested by X. Garbet 
(CEA Cadarache [6]) is to use an adapted mesh with a non-constant space step in the radial direction with mesh nodes $r_{i}$ positioned in such a way $q\left(r_{i}\right)$ is a rational number. Thus a periodic condition would become possible to settle.

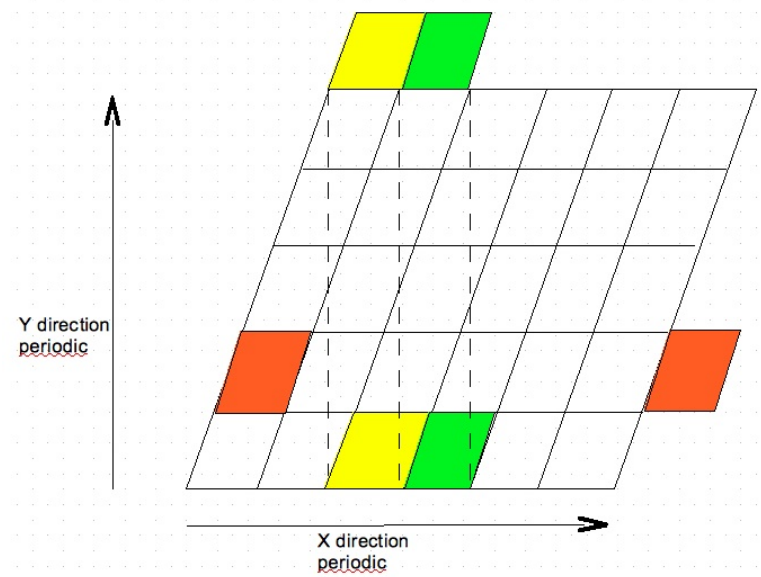

Figure 1: The domain is periodic in cartesian directions $x$ and $y$. Periodicity in $y$ direction imposes a reconnection of different mesh lines to obtain the proper periodic boundary condition.

\subsection{Remap between curvilinear and cartesian meshes}

In the context of Vlasov-Poisson like models as the Guiding-Center model or the gyrokinetic model, it can be convenient to compute the solution of the Vlasov equation using a curvilinear coordinates system and the solution of the Poisson equation on a cartesian coordinates system. Any adapted mesh will particularly be adapted to the Poisson equation, because it is roughly speaking an isotropic diffusion operator. Therefore, a cartesian mesh is the best and the simplest to design a Poisson solver. Moreover, it would decouple the computer implementation of these two solvers.

For instance, let us consider the step of the solver algorithm when the Vlasov solver needs as an input the electric potential $\Phi$ on the curvilinear mesh, whereas this quantity is computed by the Poisson solver on the cartesian mesh. We then need to remap $\Phi$ from the cartesian mesh to the curvilinear mesh. Let us consider for instance a mesh with cell centred values, such that 
the electric potential $\Phi$ values on the cartesian mesh are denoted $\Phi_{x, y}(i, j)$ at the point of coordinates $(x(i, j), y(i, j))$ with $(i, j) \in\left[1, N_{x}\right] \times\left[1, N_{y}\right]$ and has its discrete values on the curvilinear mesh denoted $\Phi_{\xi, \eta}(p, q)$ at the point of coordinates $(\xi(p, q), \eta(p, q))$ with $(p, q) \in\left[1, N_{x}\right] \times\left[1, N_{y}\right]$. We assume there exists an invertible function $f: \mathbb{R}^{2} \rightarrow \mathbb{R}^{2}$ defining the curvilinear mesh from the cartesian mesh of the following form:

$$
\xi=f_{\xi}(x, y) \text { and } \eta=f_{\eta}(x, y) .
$$

By definition of the curvilinear mesh, for any logical position $(i, j)$, the vector $\mathrm{a}(i, j)$ from the corresponding physical position on the cartesian mesh of $\Phi_{x, y}(i, j)$ to the one on the curvilinear mesh $\Phi_{\xi, \eta}(i, j)$ is

$$
\left(\begin{array}{l}
a_{x}(i, j) \\
a_{y}(i, j)
\end{array}\right)=\left(\begin{array}{l}
\xi(i, j)-x(i, j) \\
\eta(i, j)-y(i, j)
\end{array}\right)
$$

Therefore, we can use the same scheme used for the Vlasov equation, that performs a virtual advection equation of the form:

$$
\frac{\partial \Phi}{\partial t}+U \cdot \nabla_{x, y} \Phi=0
$$

with the velocity $U=a / \Delta t$ defined in such a way that, for any time step $\Delta t=t^{n+1}-t^{n}$, we obtain by following the caracteristic curves:

$$
\Phi_{\xi, \eta}(i, j)=\Phi^{n+1}(\xi, \eta)=\Phi^{n}\left(\xi-a_{x}, \eta-a_{y}\right)=\Phi^{n}(x, y)=\Phi_{x, y}(i, j),
$$

with $\Phi^{n}(x, y)$ the electric potential at a virtual virtual time $t^{n}$ and dropping arguments $(i, j)$.

The advection scheme is used with a "virtual" advection velocity in such a way quantities on the Poisson solver mesh are remapped on the Vlasov solver mesh. The same advection procedure is used to remap quantities on the Vlasov solver mesh to the Poisson solver mesh, see figure 2. In the context of the PSM scheme in which the distribution function is reconstructed by using cubic splines, this way of remaping using the advection scheme itself is obviously of the same order of accuracy as the scheme.

\section{Numerical results}

\subsection{Oblic advection}

We consider an oblic advection of a $2 \mathrm{D}$ regular sinusoidal function advected by a constant $2 \mathrm{D}$ advection field vector $\left(a_{x}=1, a_{y}=4\right)$. The initial function 


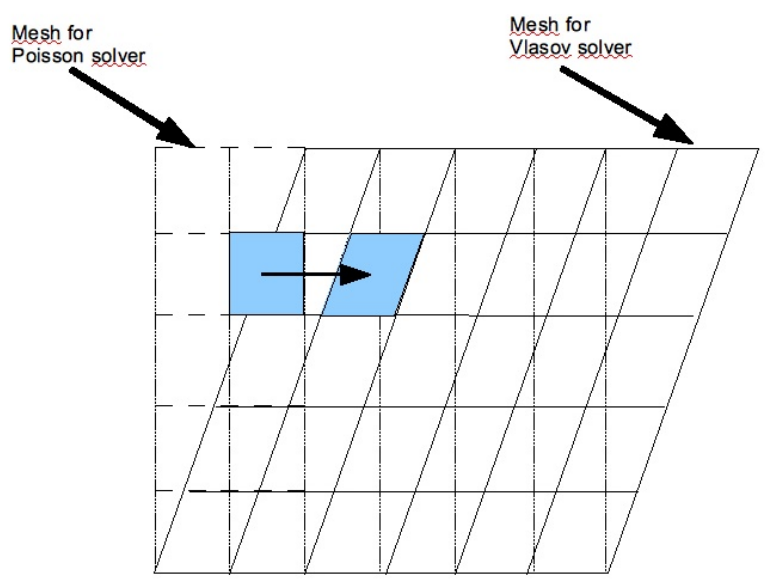

Figure 2: Remap of Poisson variables from the cartesian mesh to the curvilinear mesh and vice versa.

(see figure 3) has an obvious symmetry, considering apparent "dots" which axis is aligned with the advection direction. The domain dimensions are $[0,1]^{2}$ with a mesh of $80 \times 80$ cells. The domain is periodic in both cartesian directions $(x, y)$ and end time is calculated in such a way the final function is superimposed to the initial one after travelling ten times through the domain. The oblic curvilinear mesh is parametrized by $\alpha$, defined by the inverse of the slope of mesh lines:

$$
\xi_{1}=x-\alpha y \quad \text { and } \quad \xi_{2}=y .
$$

The issue for this benchmark is to evaluate the numerical error, function of the angle between the advection direction and the mesh lines. Figure 4 shows that when the angle between advection velocity and mesh lines is set to zero, the error is minimum. When the angle is not zero, a transversal numerical error appears because of advection velocity transversal to the mesh lines and increases as well the angle increases. Notice that error curves are not symmetric with respect to the angle value zero.

\subsection{Unstable mode for the CG model}

We use the reduced $\mathrm{CG}$ model described in the introduction with the following benchmark characteristics: 


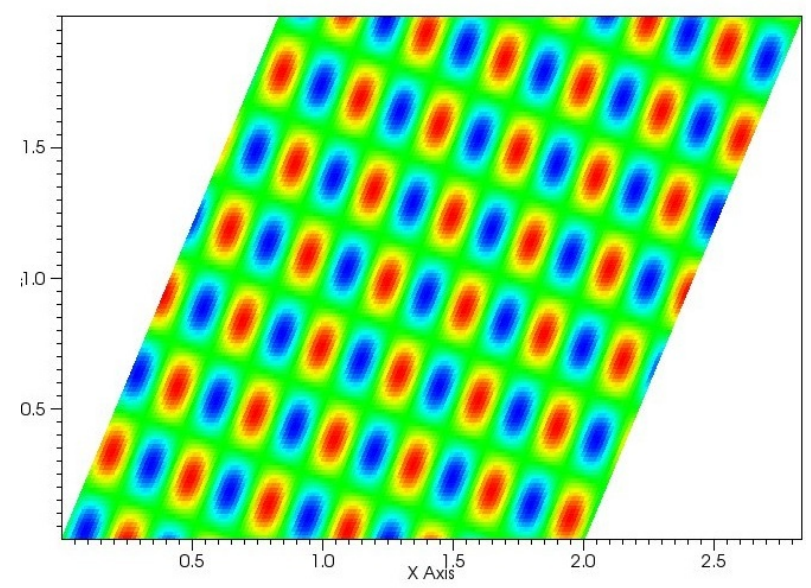

Figure 3: Initial function, advection field vector $\left(a_{x}=1, a_{y}=4\right)$ is aligned with dots but not aligned with mesh lines.

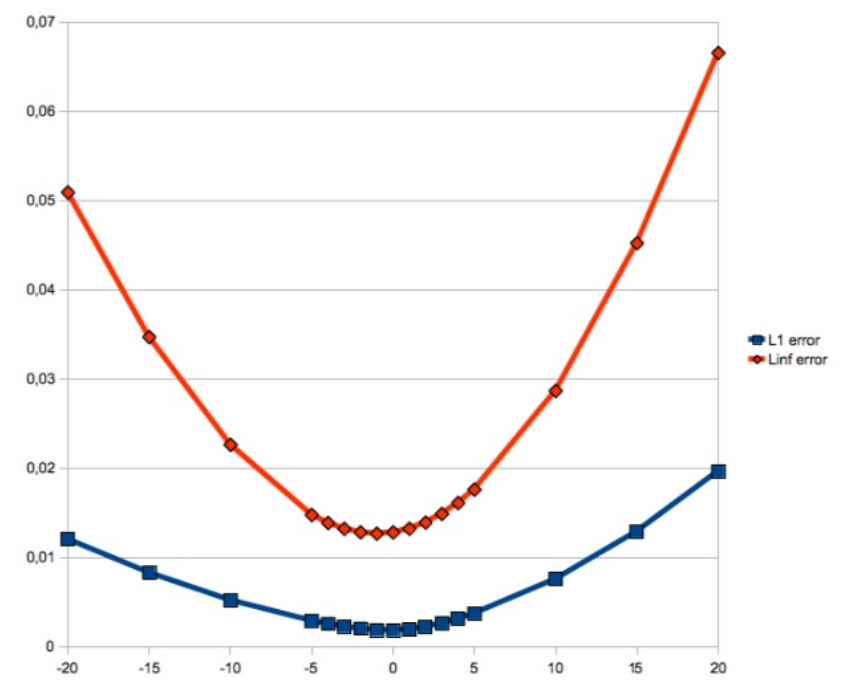

Figure 4: $L_{1}$ and $L_{\infty}$ relative error between end and initial time, function of the angle between the advection velocity vector and the mesh lines. Angle -20 corresponds to the cartesian mesh. 
- 2D periodic boundary conditions in cartesian directions $(x, y)$,

- The curvilinear mesh is made of oblic lines defined by

$$
\xi_{1}=x-\alpha y \quad \text { and } \quad \xi_{2}=y,
$$

- The unstable initial distribution function $f$ is obtained by linear modal analysis.

Using Laplace and $x$ Fourier transformations, eigenvalues $\omega$ for the CG model function of $k$ (Fourier variable in $x$ direction) may be determined through a dispersion relation. The $k$ mode growth rate is the imaginary part of $\omega$, which has to be positive to be an unstable mode, see Shoucri [9]. We use $k=1 / 2$ which is an unstable mode and has an associated growth rate $\operatorname{Im}(\omega)=1 / 2$, considering domain dimensions $(x, y)$ in $[0,2 \pi / k] \times[0,2 \pi]$ with a mesh of $128 \times 128$ cells and initial condition:

$$
f(t=0, x, y)=\sin (y)+\varepsilon \cos (k x) .
$$

We perform a qualitative comparison between PSM computations on cartesian and curvilinear meshes of a periodic-periodic instability for the CG model for two different times, one at the beginning of the nonlinear phase and the second at a late time, when most of small structures have been dissipated. The numerical results are very similar even for the late time for this complex and unstable flow.

The growth rate of the unstable mode is in good agreement in the linear phase with the expected value $\operatorname{Im}(\omega)=1 / 2$ for results on both meshes, see figure 6 .

\section{Conclusion}

The conservative PSM method with 1D directional splitting has been successfully extended for the Guiding-Center model on curvilinear coordinates, with a good agreement between cartesian and curvilinear meshes computations. Integration of the PSM method is in progress in the GYSELA code [7] [8]. The use of curvilinear coordinates for the gyrokinetic model will follow this development.

\section{References}

[1] A. J. Brizard and T. S. Hahm, Foundations of nonlinear gyrokinetic theory, Rev. Mod. Phys. 79, 421, (2007). 

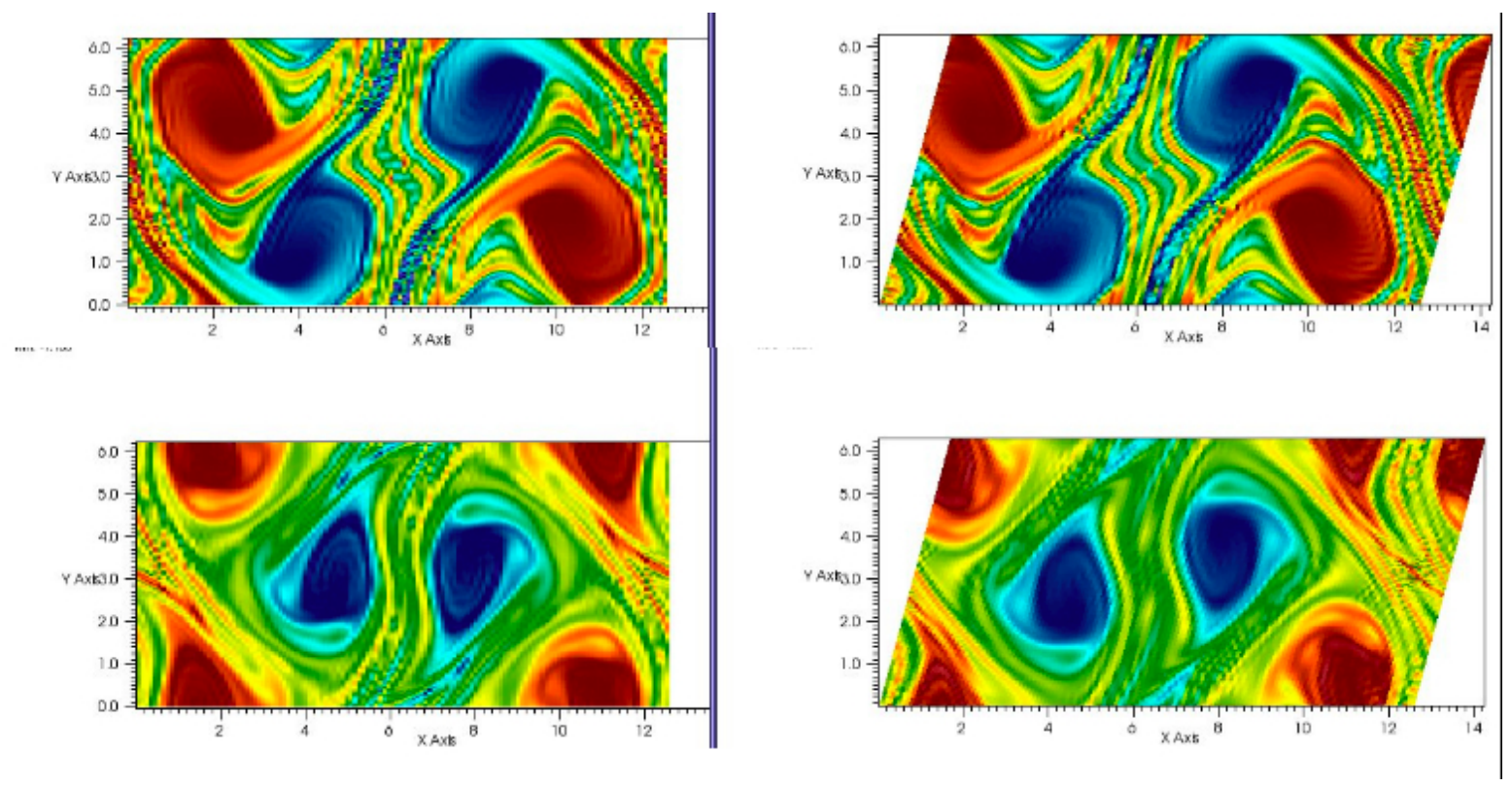

Figure 5: Comparison of cartesian (left) and curvilinear (right) computations with the PSM scheme at two different times. 


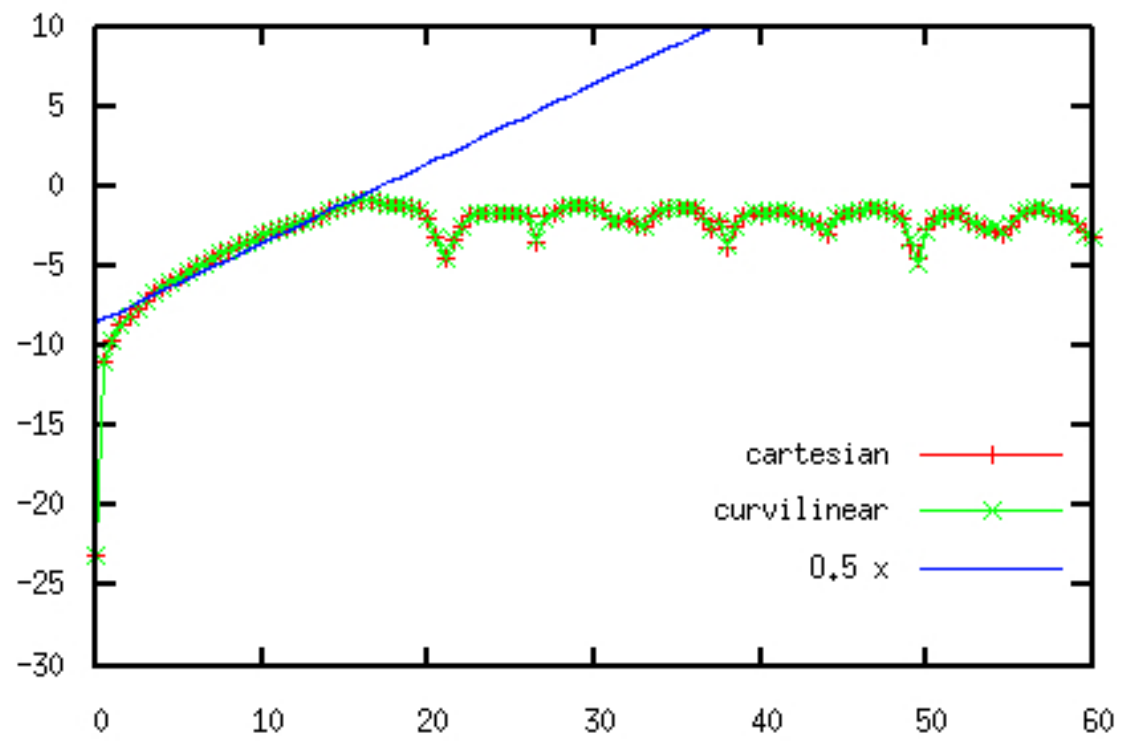

Figure 6: Comparison of cartesian and curvilinear mode evolutions versus time (plasma periods) and the theoretical growth rate in the linear phase. 
[2] N. Crouseilles, M. Mehrenberger, E. Sonnendrücker, Conservative semi-Lagrangian schemes for Vlasov equations, INRIA Report 6856, (february 2009).

[3] C.Z. Cheng, G. Knorr, The integration of the Vlasov equation in conguration space, J. Comput. Phys. 22, pp. 330-351 (1976).

[4] A. M. Dimits et al, Comparisons and physics basis of tokamak transport models and turbulence simulations, Phys. Plasmas, 7, 969, (2000).

[5] N. Crouseilles, G. Latu, E. Sonnendrücker, A Vlasov solver based on local cubic spline interpolation on patches, J. Comput. Phys., Vol. 228, pp. 1429-1446, (2009).

[6] X. Garbet, private communication 2009.

[7] V. Grandgirard et al, Global full-f gyrokinetic simulations of plasma turbulence, Plasma Phys. Control. Fus., Volume 49B, pp. 173-182 (december 2007).

[8] V. Grandgirard et al, A drift-kinetic Semi-Lagrangian 4D code for ion turbulence simulation, J. Comput. Physics, vol. 217, no2, pp. 395-423 (2006).

[9] M. Shoucri, A two-level implicit scheme for the numerical solution of the linearized vorticity equation, Int. J. Numer. Meth. Eng. 17, p. 1525 (1981).

[10] E. Sonnendrücker, J.R. Roche, P. Bertrand, A. Ghizzo, The SemiLagrangian Method for the Numerical Resolution of Vlasov Equations, J. Comput. Physics, Vol.149, No.2, pp. 201-220, (1999).

[11] M. Zerroukat, N. Wood, A. Staniforth, The parabolic spline method (PSM) for conservative transport problems. Int. J. Numer. Meth. Fluid. v11. 1297-1318, (2006).

[12] M. Zerroukat, N. Wood, A. Staniforth, Application of the parabolic spline method (PSM) to a multi-dimensional conservative semiLagrangian transport scheme (SLICE), J. Comput. Phys, Vol. 225 n.1, pp. 935-948, (2007). 